\title{
Low dose EBSD analysis of biominerals
}

Rene de Kloe ${ }^{1}$ and Matthew Nowell ${ }^{2}$

${ }^{1}$ EDAX, Tilburg, Noord-Brabant, Netherlands, ${ }^{2}$ EDAX, Riverton, Utah, United States

Electron backscatter diffraction is extensively used for orientation and microstructure characterisation of crystalline materials ranging from metals to ceramics and minerals. The application of EBSD to biominerals to date has been limited due to the often beam sensitive nature of such materials. In many cases, organic material may be present in between carbonate crystals and upon illumination with a high intensity electron beam the organic material disintegrates and the resulting damage to the material prevents successful EBSD phase and orientation analysis.

The introduction of EBSD direct electron detection allows EBSD data collection using beam currents in the $\mathrm{pA}$ range. The actual beam dose that is required depends on the backscatter coefficient, the fraction of diffracted electrons, and the accelerating voltage, but Indexable patterns can already be obtained with less than 20 electrons per pixel.

When the obtained diffracted intensity is still insufficient to get good indexing, the quality of extremely poor patterns can be enhanced by considering adjacent EBSD patterns [1] without the need to increase the beam current. This NPAR method is highly efficient because the intensity distribution and noise in the adjacent patterns is slightly different from the central target pattern. By averaging the signal with 6 neighbour patterns along a hexagonal measurement grid, the noise is effectively suppressed thereby enhancing the diffracted information (figure 1).

In this contribution a direct electron EBSD detector in combination with NPAR processing was used to investigate the crystal structure of biominerals such as the transition zone from calcite to aragonite nacre in an Atrina Pectinata shell (figure 2)

At the contact, a complex microstructure of calcite subgrains that appear correlated with aragonite grains of similar size is present. These aragonite grains first form along the edges of the calcite columns and then coalesce into full polycrystalline covers of the columns. As a final step these small aragonite crystals are then covered by equiaxed planar aragonite crystals that form the nacre structure that coats the inside of the shell. 

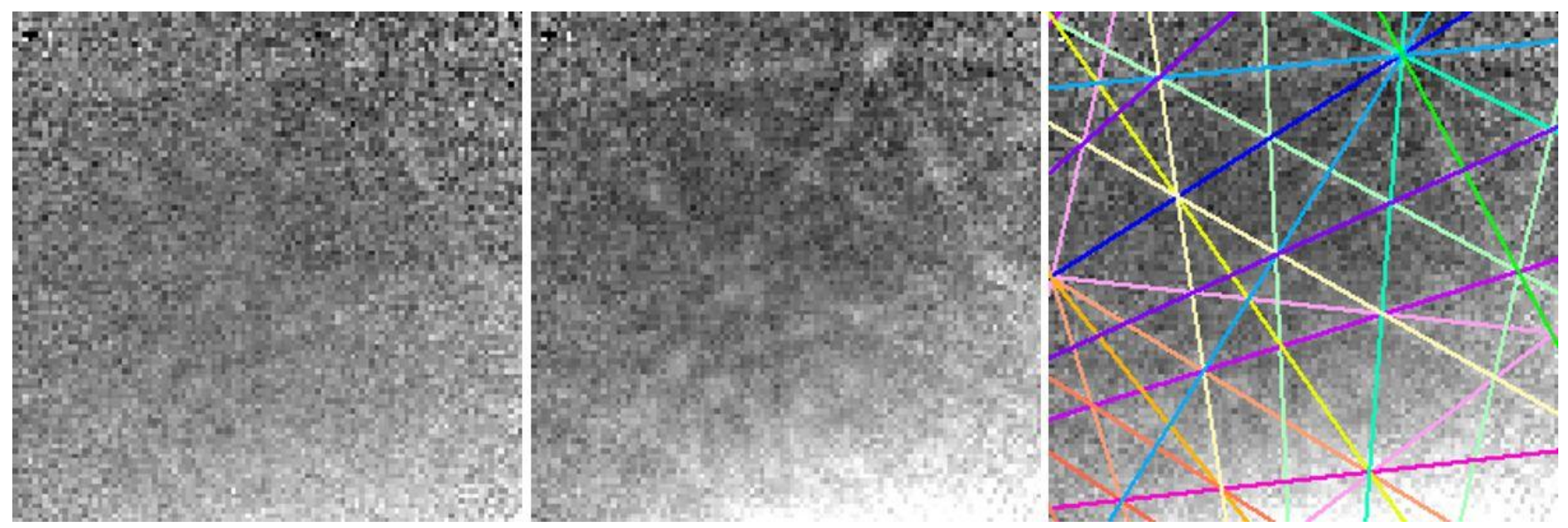

Figure 1. Extremely weak aragonite diffraction pattern as measured, after NPAR processing and with indexing solution
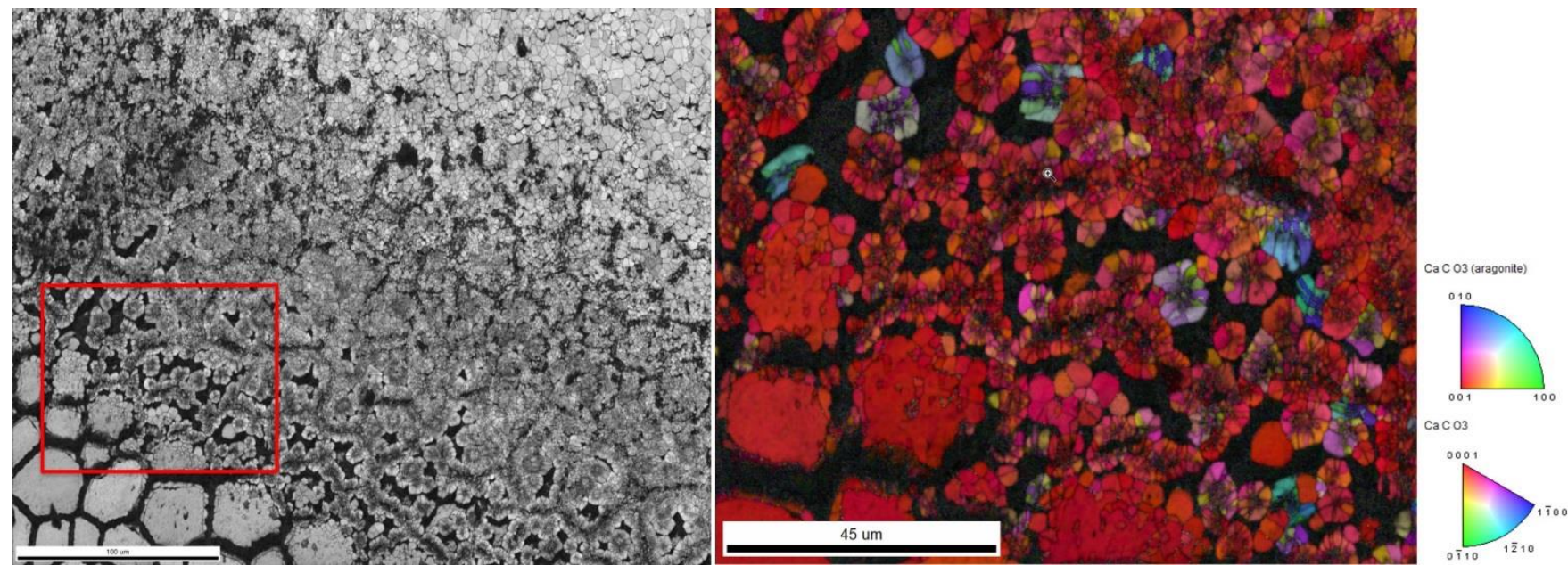

Figure 2. EBSD Image Quality map showing the transition zone from columnar calcite (lower left) to planar aragonite nacre (top right). The detail Inverse Pole Figure orientation map illustrates the grain microstructure directly at the calcite-aragonite contact

References

[1] Ultramicroscopy, Volume 159, Part 1, December 2015, Pages 81-94 